\title{
UN ANÁLISIS DEL COMPORTAMIENTO CÍCLICO DE LAS COOPERATIVAS Y SOCIEDADES LABORALES ESPAÑOLAS Y DE SU RELACIÓN CON LA ACTIVIDAD ECONÓMICA
}

\author{
POR \\ Mercé SALA RÍOS, \\ Mariona FARRÉ PERDIGUER y \\ Teresa TORRES SOLÉ ${ }^{1}$
}

\section{RESUMEN}

Existe un amplio debate acerca del papel que ha jugado y juega la Economía Social en el proceso de desarrollo y de creación de empleo estable. En dicho debate resulta de interés conocer cuáles son las características que definen las fases cíclicas de las empresas de participación y de su relación con el ciclo económico. El comportamiento cíclico de las empresas de participación respecto al conjunto de la actividad económica marcará sin duda sus efectos sobre el desarrollo y el empleo. El trabajo que presentamos se mueve en esta línea de investigación, el análisis se centra en la economía española y en las dos principales figuras de la Economía Social, las cooperativas y las sociedades laborales. Los principales objetivos que se persiguen son los dos siguientes. En primer lugar, caracterizar las fases cíclicas del número de empresas y del empleo de las cooperativas y de las sociedades laborales y compararlas con las fases cíclicas de la actividad económica española. En segundo lugar, analizar cómo las diferencias/similitudes detectadas en las características de las fases afectan al nivel de sincronización con el ciclo económico. El periodo que entra en el estudio es desde el primer trimestre de 1995 (1995:1) hasta el segundo de 2013 (2013:2). Cabe señalar que un elemento novedoso dentro del análisis propuesto es el hecho de trabajar por separado las cooperativas y las sociedades laborales. La literatura predominante o bien se centra en el

\footnotetext{
${ }^{1}$ Departamento de Economía Aplicada. Universidad de Lleida. Direcciones de correo electrónico: mercesa@econap.udl.cat; mariona.farre@econap.udl.cat; torres@econap.udl.cat
}

REVESCO No 115 - Segundo Cuatrimestre 2014 - ISSN: 1885-8031 - www.ucm.es/info/revesco

http://dx.doi.org/10.5209/rev_REVE.2014.v115.45279

Fecha de recepción: 11/11/2013

Fecha de aceptación: 29/04/2014 
análisis global de la Economía Social o bien da mayor protagonismo al ámbito de las cooperativas.

Según los resultados obtenidos, las sociedades laborales muestran un comportamiento pro-cíclico importante tanto en cuanto a su creación como a su desaparición, comportamiento que acaba trasladándose, aunque con menor intensidad, a la creación o destrucción de empleo. Por su parte las cooperativas evolucionan de forma bastante desvinculada de la actividad económica. La creación y destrucción de cooperativas y su empleo alcanzan valores relativamente bajos de sincronización con el ciclo económico.

Palabras clave: ciclo económico, fluctuaciones cíclicas, expansión, contracción, sincronización cíclica.

Claves Econlit: E320; J540; M200; P130

\title{
SPANISH COOPERATIVE CYCLIC BEHAVIOR AND LABOR COMPANIES. WHAT IS ITS RELATION TO ECONOMIC ACTIVITY?
}

\begin{abstract}
There is a wide debate about the role that the Social Economy plays in the process of development and creation of stable employment. In this debate is important to analyze what characteristics define the cyclical phases of the worked-own firms and their relationship with the business cycle. The cyclical behavior of the worked-own firms in relation to the business cycle will influence in the economic development and employment. This work revisits this question on the Spanish economy. We present a comparative empirical examination centered on cooperatives and on labor societies. The main objectives are two. Firstly, we characterize the cyclical phases of the number of firms and of the employment, both in cooperatives and in labor societies. We compare these characteristics with those of the business cycle. Secondly, we analyze how the presence or absence of differences detected in this comparison affects to the degree of synchronization with the business cycle. The period studied is from the first quarter of 1995 (1995:1) to the second of 2013 (2013:2). It is noteworthy that an innovation in our analysis is that we work separately cooperatives and labor societies. Most of the literature focuses on either the overall analysis of the social economy or gives greater prominence to cooperatives.
\end{abstract}

REVESCO No 115 - Segundo Cuatrimestre 2014 - ISSN: 1885-8031 - www.ucm.es/info/revesco 
According to the results, labor societies show a significant pro-cyclical behaviour, both in the creation and in the extinction of firms. This behaviour is transferred with less strongly, to the creation or destruction of employment. Meanwhile, cooperatives evolve quite decoupled to the economic activity. The creation and destruction of cooperatives and their employment reach a low level of synchronization with the business cycle.

Keywords: business cycle, cyclical fluctuations, expansion, contraction, cyclical synchronization.

\section{INTRODUCCIÓN}

En los estudios sobre Economía Social encontramos numerosos trabajos que sustentan el papel de las empresas sociales en el proceso de desarrollo económico y social del territorio. Melián y Campos (2010) ven una relación directa entre entidades de Economía Social y desarrollo local. Según los autores, en tiempos de crisis la Economía Social da la oportunidad a los emprendedores de nuevas iniciativas que contribuyen al mantenimiento del empleo. El emprendedor social al incorporar en su proyecto objetivos empresariales y fines sociales genera empleo estable, profesionaliza la gestión y fomenta el desarrollo local.

Por su parte Tarazona y Albors (2005) identifican tres características de las empresas e instituciones de la Economía Social que las relacionan directamente con el desarrollo local. En primer lugar, su dimensión local, lo que incrementa su compromiso con el territorio donde se ubican y les confiere proximidad a los colectivos que las necesitan. En segundo lugar, su carácter social que les lleva a priorizar el trabajo por encima del capital y a crear empleos más estables. En tercer lugar, la participación de los trabajadores en el ámbito directivo asegura su motivación, al tiempo que el carácter empresarial de las instituciones asegura la profesionalización de la gestión.

Asimismo, es un sector al que se le reconoce la capacidad de combinar aspectos económicos con temas de inserción social. Monzón y Chaves (2012) indican que la Economía Social ha sido uno de los sectores que ha ayudado a la estabilidad laboral porque además de crear nuevos puestos de trabajo y de conservar los existentes en los sectores y negocios en crisis o amenazados por el cierre, ha sido una vía de acceso al empleo para los grupos desfavorecidos y las personas excluidas socialmente. 
Según Grávalos y Pomares (2001a) las crisis de las últimas décadas generaron una serie de hechos que explican el auge de las empresas de participación. Entre ellos destacan por un lado, la transformación de empresas tradicionales en empresas de participación con el fin de mantener los puestos de trabajo. Por otro lado, ante el aumento del desempleo, los parados reaccionan y deciden luchar contra el paro estructural creando sus propias empresas. Monzón (1991) va más allá y reconoce a las empresas de la Economía Social la capacidad de resolver problemas sociales que las empresas públicas o privadas no han podido resolver satisfactoriamente.

Calderón y Calderón (2012a) se ocupan de la calidad en el empleo de las cooperativas, sociedades laborales y centros especiales de empleo, como entidades representantes de la Economía Social en España, en un marco de fuerte crisis económica como es el año 2009. Sus conclusiones revelan que estas entidades gozan de mayor calidad en el empleo gracias a elementos como la igualdad de género, mayor estabilidad laboral, mayor flexibilidad para ajustarse vía horas trabajadas más que vía número de trabajadores y la no discriminación o inclusión. El estudio de Lejarriaga et al. (2013) reconoce estas virtudes en las empresas de trabajo asociado, sin embargo, su estudio empírico centrado en el emprendimiento entre jóvenes universitarios, pone de manifiesto el desconocimiento por parte de este colectivo de su existencia. Es por ello que consideran de vital importancia el asesoramiento técnico y una labor de difusión de estas formas jurídicas como fórmulas alternativas de emprender.

Según lo dicho resulta de interés analizar el papel de la Economía Social en la evolución del ciclo de la actividad económica. ¿Cuáles son las características que definen las fases cíclicas de las empresas sociales? Y en función de estas características ¿Qué grado de sincronización tiene su ciclo con el del conjunto de la actividad económica? Estas son las preguntas a las que daremos respuesta en este estudio, centrándonos en la economía española y reduciendo el ámbito de la Economía Social a las cooperativas y las sociedades laborales.

Según la Ley 5/2011 de 29 de marzo de Economía Social, en España la Economía Social engloba el conjunto de actividades económicas y empresariales que en el ámbito privado llevan a cabo aquellas entidades que persiguen el interés general económico, social o ambos. Las entidades que forman la Economía Social son las cooperativas, las sociedades laborales, las mutualidades, los centros especiales de empleo, las empresas de inserción, las cofradías de pescadores, las asociaciones y las fundaciones. Como se ha dicho, en el estudio que desarrollaremos nos centraremos en las cooperativas y en las sociedades laborales. Los 
motivos de esta decisión son por una parte, porque son las dos principales figuras de la Economía Social, entre las dos representan el 79,17\% del número de empresas e instituciones de la Economía Social (50,37\% las cooperativas y 28,80\% las sociedades laborales) y aportan el $91,59 \%$ del empleo $(73,3 \% \text { y 18,29\%, respectivamente })^{2}$. Por otra parte, porque resultan adecuadas para compararlas con el conjunto de la actividad económica porque de todas las instituciones que integran la Economía Social, su actividad es la que más se mueve en la línea y bajo los objetivos de una empresa privada.

Del mismo modo que hemos nombrado estudios que se ocupan de la Economía Social en su conjunto, también encontramos numerosos estudios que analizan los mismos temas pero centrándose sobretodo en las cooperativas ${ }^{3}$. Staber (1993) apunta que en ocasiones se sugiere que las cooperativas son lo suficientemente flexibles tanto para desenvolverse en sectores industriales poco atractivos como para explotar oportunidades en sectores más atractivos y emergentes. La evidencia empírica confiere en algunos casos, un comportamiento contracíclico a las cooperativas otorgándoles un papel fundamental a la hora de absorber mano de obra en un escenario de destrucción de empleo. Estos análisis concluyen que las cooperativas sobreviven independientemente de las condiciones económicas adversas ${ }^{4}$.

Grávalos y Pomares (2001a) para la economía española en el periodo1986-1995 o Demoustier (2000) para Europa en el periodo 1991-1995, indican que las cooperativas ejercen un efecto refugio para el empleo en épocas de crisis, confirmándose un comportamiento contra-cíclico ${ }^{5}$. Díaz y Marcuello (2010) estudian los años 1995 a 2008 y concluyen que ante aumentos y disminuciones del PIB el empleo creado por las cooperativas se modifica en menor medida que el empleo total, aunque para estos años no se puede derivar un efecto refugio como el defendido por los autores anteriores. Román (2010) realiza un diagnóstico del comportamiento de las cooperativas desde inicios de los años cuarenta hasta inicios del actual siglo (1942-2002), el objetivo es conocer si los ciclos de creación de las cooperativas guardan relación con las etapas de expansión o contracción. Según sus resultados, en períodos de dificultades económicas las cooperativas agrícolas y de consumo mostraron mayor resistencia

\footnotetext{
${ }^{2}$ Los datos se refieren al año 2012 en lo relativo al número de empresas e instituciones y al año 2011 en lo relativo a empleo. La fuente es la Confederación Empresarial de Economía Social (CEPES):

http://www.cepes.es/tipos_de_entidades.

${ }^{3}$ Para una revisión de la literatura no sólo de las cooperativas sino del conjunto de la Economía Social véase por ejemplo Clemente et al. (2008; 2009).

${ }^{4}$ Díaz y Marcuello (2010) o Calderón y Calderón (2012b) presentan diversos trabajos en los que se establece una relación inversa entre el sector cooperativo y el ciclo económico.

${ }^{5}$ Grávalos y Pomares (2001b) llegan a conclusiones semejantes para las sociedades laborales españolas y dentro del mismo periodo (1986-1995).
} 
que otras sociedades mercantiles, aunque también respondieron con rapidez a los estímulos de las etapas de expansión.

Monzón (2012) señala que en España y dentro del escenario de recesión iniciado en el año 2007, las cooperativas son un ejemplo de crecimiento inteligente, sostenible e integrador. Han demostrado un espíritu innovador en la medida que están lanzando nuevos productos al mercado de forma exitosa y pionera. Además, según el autor, están mostrando también capacidad innovadora en la organización lo que unido a una gestión más participativa y al mayor compromiso de los trabajadores, les permite resistir más a la crisis y les confiere ciertas ventajas competitivas. En esta misma línea, Calderón y Calderón (2012b) argumentan que en la actual crisis la menor destrucción de empleo de las cooperativas en relación al conjunto de la economía se debe al proceso de transición de trabajadores que proceden de otras empresas hacia las cooperativas y/o a la integración de desempleados, ya sea como trabajadores o como socios.

No todas las investigaciones llegan a estas mismas conclusiones. Así, también encontramos estudios en los que se subraya que las cooperativas se crearon y sobrevivieron al margen de las condiciones generales de la economía. En este sentido el trabajo empírico de Staber (1993), contrariamente a la asunción generalmente extendida, señala que el comportamiento de las cooperativas no tiene un carácter contra-cíclico ${ }^{6}$. Ben-Ner (1988) sugiere que, según el análisis comparativo entre empresas de participación y empresas privadas realizado, se podría llegar a generalizar que las tasas de creación y desaparición de las empresas de participación siguen un comportamiento contra-cíclico. Sin embargo, este patrón se ve en parte compensado porque se observa que en épocas expansivas también se crean empresas de participación y su desaparición se muestra vulnerable a las fases contractivas. Para Russell y Hanneman (1992) o Pérotin (2006), la entrada o creación de cooperativas es contra-cíclica en cambio, encuentran menor evidencia en que también lo sea la salida o desaparición.

Clemente et al. $(2008,2009)$ en un estudio que abarca las cooperativas y las sociedades laborales de la economía española en el periodo 1999-2007, analizan el proceso de convergencia por Comunidad Autónoma y observan que estas empresas no presentan la misma tendencia que el conjunto de la economía. Los autores evidencian que para la

\footnotetext{
${ }^{6}$ En el artículo de Staber (1993) se recogen otros trabajos empíricos en desacuerdo con el comportamiento contra-cíclico descrito.
} 
economía en su conjunto las Comunidades Autónomas tienden a converger mientras que la Economía Social acentúa las divergencias, desarrollándose más en las Comunidades Autónomas con un entorno económico de mayor nivel de renta per capita y de mayor nivel de empleo.

El trabajo que presentamos se mueve en las líneas de estudio descritas. En este sentido, pretende contrastar el comportamiento cíclico de las cooperativas y de las sociedades laborales en relación al conjunto de la actividad económica española. Dos son los principales objetivos. En primer lugar, caracterizar las fases cíclicas del número de empresas y del empleo de las cooperativas y de las sociedades laborales y compararlas con las fases cíclicas de la actividad económica. En segundo lugar, analizar cómo las diferencias/similitudes detectadas en las características de las fases afectan al nivel de sincronización con el ciclo económico. Los resultados permitirán detectar la existencia de un comportamiento pro/contracíclico de las cooperativas y las sociedades laborales de la economía española dentro de los años de estudio. También nos interesará ver la incidencia de la crisis iniciada en 2007 en la sincronización de los ciclos.

Cabe señalar que un elemento novedoso dentro del análisis propuesto es el hecho de trabajar por separado las cooperativas y las sociedades laborales. La literatura predominante o bien se centra en el análisis global de la Economía Social o bien da mayor protagonismo al ámbito de las cooperativas. En este estudio, los resultados a los que lleguemos nos aportarán evidencia empírica del comportamiento cíclico de las cooperativas y de las sociedades laborales, de sus diferencias y de su relación con el ciclo económico. En nuestra opinión, serán un instrumento valioso para posteriores investigaciones en las que se desee profundizar en la explicación de las causas que se esconden detrás de los comportamientos aquí revelados.

El trabajo se organiza de la siguiente forma. En el segundo apartado se presenta la metodología seguida a lo largo del estudio. Así, se recogen las fuentes estadísticas, el proceso para determinar las fases cíclicas y los indicadores para caracterizar el ciclo y analizar la sincronización. El tercer apartado se ocupa de los resultados y el cuarto expone las principales conclusiones.

\section{METODOLOGÍA}

El indicador que se ha utilizado para aproximar las fluctuaciones cíclicas de la economía española es el componente cíclico del PIB real. Para construirlo hemos trabajado 
con los datos del PIB real trimestral corregido de estacionalidad. La fuente estadística ha sido el INE. Por lo que respecta a los datos de la Economía Social, se analiza el comportamiento cíclico del número de cooperativas y de sociedades laborales y de su empleo. Los datos se han obtenido de los Datos Estadísticos de Economía Social del Ministerio de Empleo y Seguridad Social. Son datos trimestrales del número de cooperativas (CS) y sociedades laborales (SL), dadas de alta en la Seguridad Social y de su número de trabajadores. El periodo que entra en el estudio es desde el primer trimestre de 1995 (1995:1) hasta el segundo de 2013 (2013:2).

El componente cíclico se ha obtenido aplicando el filtro de Hodrick-Prescott (HP) a las series trimestrales. La elección de HP se ha realizado básicamente porque al ser simétrico no produce movimientos de fase, porque aproxima bien a un filtro ideal cuando se utiliza un $\lambda=1600$ para datos trimestrales y porque es un método operacional.

\subsection{Determinación de las fases cíclicas y caracterización}

Siguiendo el enfoque de Burns y Mitchel (1946), que es uno de los trabajos que mayor reconocimiento ha tenido en el ámbito de las fluctuaciones cíclicas, entendemos que un ciclo consiste en fluctuaciones de la actividad económica en fases expansivas y contractivas que se producen de forma recurrente. Según esta terminología, se define un pico (peak) como el momento en el tiempo inmediatamente anterior a una disminución de la actividad económica y un valle (trough) como el momento previo al crecimiento de la actividad económica, ambos se conocen como puntos de giro o puntos de inflexión (turning points). La contracción es el lapso que media entre el pico inicial $\left(\mathrm{P}_{\mathrm{i}}\right)$ y el valle $(\mathrm{V})$ y la expansión es el lapso que media entre el valle $(\mathrm{V})$ y el pico final $\left(\mathrm{P}_{\mathrm{f}}\right)$. La duración del ciclo es el tiempo que transcurre entre un pico y el siguiente o entre un valle y el siguiente.

A partir del trabajo de Burns y Mitchell (1946), encontramos abundante literatura centrada en el estudio de las características específicas de los ciclos económicos que se apoya al menos en dos diferentes metodologías. La primera, de carácter no-paramétrico, para definir los puntos de giro recurre a algoritmos, el más tradicional el de Bry y Boschan (1971) que Harding y Pagan (2002) adaptan para datos trimestrales. La segunda, se basa en el modelo de cambio de régimen propuesto por Hamilton (1989) y se ha desarrollado mediante técnicas econométricas ${ }^{7}$.

\footnotetext{
${ }^{7}$ Gadea et al. (2012) presentan y referencian trabajos en los que se discuten ambas metodologías.
} 
Al trabajar con datos trimestrales, para obtener los picos y valles seguimos a Harding y Pagan (2002). Las fases del ciclo se han identificado mediante el software BUSY desarrollado por la Comisión Europea (Fiorentini y Planas, 2003). Las reglas fundamentales son tres. En primer lugar, un máximo/mínimo local se define como el punto más alto/bajo entre los dos trimestres predecesores y antecesores a su posición. Es decir $\mathrm{y}_{\mathrm{t}}$ es un pico en el tiempo $\mathrm{t}$ si $\mathrm{y}_{\mathrm{t}}$ es el $\max \left(\mathrm{y}_{\mathrm{t}-2} \ldots \mathrm{y}_{\mathrm{t}+2}\right)$ y es un valle si $\mathrm{y}_{\mathrm{t}}$ es el $\min \left(\mathrm{y}_{\mathrm{t}-2} \ldots . . \mathrm{y}_{\mathrm{t}+2}\right)$. En segundo lugar, un ciclo siempre debe completarse, por lo que un pico debe ir seguido de un valle y viceversa, con una duración mínima de la fase cíclica de tres trimestres. Por último, la duración mínima de un ciclo completo es de cinco trimestres ${ }^{8}$.

Una vez identificados los puntos de giro, las fases de expansión y contracción y conocidos los ciclos completos, caracterizaremos el ciclo en términos de duración, amplitud y retardos. La duración (D) de la contracción (expansión) es el número de trimestres que transcurren entre el pico del ciclo y su valle final (valle del ciclo y su pico final). Pudiéndose obtener de aquí la duración media de cada fase. La amplitud de una contracción (expansión) se calcula como el porcentaje de variación, en valor absoluto, entre el valor en el valle (pico) y el pico anterior (valle). La amplitud estima las ganancias (pérdidas) en términos de actividad, número de empresas o de trabajadores, según la variable que estemos observando, en las fases de expansión (contracción). El retardo (adelanto) es la media del número de retardos (adelantos) en los puntos de giro que pueden contabilizarse entre una serie y la serie que se toma de referencia.

\subsection{Sincronización cíclica}

Las diferencias/similitudes en las características de las fases afectan a la sincronización temporal de los ciclos. El análisis de la sincronización se iniciará calculando un indicador para todo el período. De acuerdo con Harding y Pagan (2002), obtendremos el índice de concordancia (I) que entiende la sincronización como la proporción de tiempo en la que dos variables $(i, j)$ se encuentran en la misma fase:

$$
I_{i j}=T^{-1}\left[\sum_{t=1}^{T}\left(S_{i t} S_{j t}\right)+\sum_{t=1}^{T}\left(1-S_{i t}\right)\left(1-S_{j t}\right)\right]
$$

\footnotetext{
${ }^{8}$ Como indican Harding y Pagan (2002) la duración mínima de 15 meses establecida por Bry y Boschan (1971) es compatible con 4 o 5 trimestres, dependiendo del mes en el que se produzca el punto de quiebre y de las magnitudes relativas de los meses dentro del trimestre. Es por ello que se han calculado los puntos de giro con 4 y con 5 trimestres sin que ello haya variado los puntos de giro, con lo que los resultados se presentan a partir de 5 trimestres.
} 
donde $S_{\mathrm{it}} / \mathrm{S}_{\mathrm{jt}}$ es una variable binaria que toma el valor 1 cuando $\mathrm{i} / \mathrm{j}$ está en expansión y cero cuando está en recesión y T es el número de observaciones. El índice se mueve entre uno y cero. La unidad indica perfecta concordancia y cero perfecta inexistencia de concordancia.

El índice nos proporciona una información fácil de interpretar pero no nos dice nada acerca de si los co-movimientos son estadísticamente significativos y si el grado de asimetría entre ambos puede llegar a ser contra-cíclica. Precisamente para salvar esta dificultad estimaremos el coeficiente de correlación $(\rho)$ entre $S_{\text {it }}$ y $S_{j t}$, usando el método generalizado de momentos según la metodología propuesta por Harding y Pagan (2006). Partimos de la condición de los momentos:

$E\left[\sigma s_{i}^{-1}\left(S_{i t}-\mu s_{i}\right) \sigma s_{j}^{-1}\left(S_{j t}-\mu s_{j}\right)-\rho s\right]=0$

donde $\sigma \mathrm{s}$ y $\mu$ s son respectivamente la media y la desviación típica de las series $S_{\text {it }} \mathrm{y}$ $\mathrm{S}_{\mathrm{jt}}$. El estimador que se genera es:

$\frac{1}{T} \sum_{t=1}^{T}\left[\hat{\sigma} s_{i}^{-1}\left(S_{i t}-\hat{\mu} s_{i}\right) \hat{\sigma} s_{j}^{-1}\left(S_{j t}-\hat{\mu} s_{j}\right)-\hat{\rho} s\right]=0$

El proceso de estimación utilizado es el de Newey y West (1987) (HAC) y pesos de Bartlett que resulta consistente a la presencia de correlación serial y heteroscedasticidad. La significancia estadística puede contrastarse entonces usando la t-ratio. Una t-ratio significativa y positiva indica sincronización pro-cíclica. En cambio, una t-ratio significativa y negativa indica asimetría contra-cíclica

Con los cálculos anteriores tendremos una visión conjunta del grado de sincronización dentro del período. Para poder desglosar temporalmente la sincronización cíclica calcularemos un índice de asimetría que cuantifica la tendencia de la sincronización de los ciclos (Larsson et al., 2009 quién cita a Hassler, 2003). Se normalizará el componente cíclico de las series $(\mathrm{Y})$, mediante la desviación estándar $(\sigma)$ : $\mathrm{Y}^{*}=\mathrm{Y} / \sigma$. El índice entre dos variables se define como:

$$
I A_{i j t}=\left|Y_{i t}^{*}-Y_{j t}^{*}\right|
$$


$\mathrm{Si}$ el índice es cero las dos variables presentan plena sincronización en los ciclos, por el contrario, si el índice es elevado ambas variables muestran asimetrías importantes en sus ciclos.

\section{RESULTADOS}

Iniciamos el análisis de los resultados caracterizando las fases cíclicas. La tabla 1 recoge los puntos de giro. El PIB, el número y el empleo de SL presentan dos ciclos completos de valle a valle. Las CS en cambio, alcanzan un único ciclo completo de valle a valle.

Tabla 1: Turning Points PIB, número de empresas y número de trabajadores

\begin{tabular}{|c|c|c|c|c|c|}
\hline \multicolumn{2}{|c|}{ PIB } & \multicolumn{2}{|c|}{ Número CS } & \multicolumn{2}{|c|}{ Número SL } \\
\hline \multirow[t]{2}{*}{ Pico } & Valle & Pico & Valle & Pico & Valle \\
\hline & $1997: 3$ & $1997: 2$ & $2000: 2$ & & 1997:3 \\
\hline 2002:4 & $2004: 3$ & & & 2002:2 & 2004:4 \\
\hline $2007: 4$ & 2009:3 & $2007: 4$ & 2009:4 & $2007: 4$ & 2009:4 \\
\hline & & \multicolumn{2}{|c|}{ Trabajadores CS } & \multicolumn{2}{|c|}{ Trabajadores SL } \\
\hline & & Pico & Valle & Pico & Valle \\
\hline & & 1999:4 & $2002: 3$ & & $1997: 4$ \\
\hline & & & & 2001:2 & $2002: 4$ \\
\hline & & $2007: 4$ & $2010: 2$ & $2007: 1$ & $2009: 4$ \\
\hline & & 2012:4 & & & \\
\hline
\end{tabular}

\subsection{Caracterización de las fases cíclicas}

Nos ocuparemos en primer lugar de las características de las fases cíclicas del número de CS y SL, recogidas en la tabla 2. Centrándonos en las fases expansivas, vemos que las CS son las que presentan mayor duración media, 30 trimestres frente a los 15,5 de las SL y a los 17 del PIB. En cambio, la amplitud media del número de CS es relativamente menor que la amplitud del número de SL. Esto indica que en épocas de expansión las ganancias medias en número de SL exceden las del número de CS.

En ambos tipos de empresa, los retardos con respecto al PIB son mínimos, lo que en principio implica que las tres variables alcanzan los picos de sus fases de forma bastante 
sincrónica. Sin embargo, si analizamos los puntos de giro de la tabla 1 vemos que si bien es lo que se produce entre el PIB y las SL, no es así para las CS. El mínimo retardo entre el PIB y las CS deriva de un único pico que puede compararse y que coincide en el tiempo (2007:4). El pico del PIB (2002:4) no puede compararse con ningún pico de CS, y el pico (1997:2) de CS le ocurre exactamente lo mismo. Por tanto, el dato esconde diferencias importantes en las fases expansivas de las CS y la actividad económica.

En las fases contractivas vemos que, tanto para las CS como para las SL, la duración media es superior a la de la actividad económica (10; 9 y 7 trimestres respectivamente). Según los datos de la amplitud, son las SL las que experimentan menos pérdidas en las fases contractivas, $148,38 \%$ frente al $165,59 \%$ de las CS. Así podemos indicar que en sus fases contractivas, las CS pierden mayor número de empresas que las que pierden las SL en las suyas.

El retardo medio de las CS respecto del PIB es de 6 trimestres, lo cual es una cifra significativa. Todo lo contrario de lo que ocurre en el caso de las SL, aquí el valle de las fases contractivas se alcanza con un retardo medio de menos de un trimestre respecto al PIB. Como ocurría en la expansión, las cifras parecen indicar que el proceso contractivo del número de CS se produce con cierta desvinculación respecto de la evolución de la actividad económica de la economía española, afirmación que no puede extenderse al caso de las SL.

Tabla 2: Duración, Retrasos y Amplitud PIB y del número de CS y de SL

\begin{tabular}{|l|c|c|c|c|c|c||}
\cline { 2 - 7 } \multicolumn{1}{c||}{} & \multicolumn{3}{c|}{ Expansión } & \multicolumn{3}{c||}{ Contracción } \\
\cline { 2 - 8 } & $\begin{array}{c}\text { Retardo } \\
\text { medio } \\
\text { (trimestres) }\end{array}$ & $\begin{array}{c}\text { Duración media } \\
\text { (trimestres) }\end{array}$ & $\begin{array}{c}\text { Amplitud } \\
\text { media } \\
(\%)\end{array}$ & $\begin{array}{c}\text { Retardo } \\
\text { medio } \\
\text { (trimestres) } \\
1\end{array}$ & $\begin{array}{c}\text { Duración } \\
\text { media } \\
\text { (trimestres) }\end{array}$ & $\begin{array}{c}\text { Amplitud } \\
\text { Media } \\
\text { Valor absoluto } \\
(\%)\end{array}$ \\
\hline PIB & 17 & 213,65 & & 7 & 233,51 \\
\hline Número CS & 0 & 30 & 248,01 & 6 & 10 & 165,59 \\
\hline Número SL & -1 & 15,50 & 262,24 & 0,67 & 9 & 148,38 \\
\hline \hline
\end{tabular}

(1) CS y Laborales respecto a PIB. + (-) implica retardo (adelanto)

Fuente: BUSY y elaboración propia

Pasemos a analizar el comportamiento del empleo. En la tabla 3 se recogen las características de las fases cíclicas del número de trabajadores de las CS y de las SL. Si comparamos las fases expansivas del empleo con el PIB, vemos que es este el que tiene una mayor duración media, 17 trimestres (tabla 2) frente a los 15,5 que presenta el empleo tanto de las CS como de las SL (tabla 3). La amplitud media del empleo es relativamente más 
importante en las SL que en las CS. Esto indica que en épocas de expansión la capacidad de generar puestos de trabajo de las SL excede al de las CS. Los datos relativos a los retardos de la tabla 3 apuntan a que en la expansión, el empleo se adelanta al PIB, así, la capacidad de crear empleo de las CS y de las SL se agota antes que la capacidad de la economía de generar crecimiento económico.

En las fases contractivas, la duración media de CS y SL es superior a la de la actividad económica (10,5; 8,5 y 7 respectivamente). Según la amplitud, son las CS las que tienen menor pérdida de empleo. El retardo medio revela que de nuevo las CS y las SL se adelantan a la actividad económica a la hora de alcanzar los valles de las fases contractivas. Las empresas sociales recuperan la capacidad de generar empleo cuando la actividad económica todavía no ha alcanzado la fase expansiva.

Tabla 3: Duración, Retrasos y Amplitud número de trabajadores de CS y de SL

\begin{tabular}{||c|c|c|c|c|c|c||}
\cline { 2 - 7 } \multicolumn{1}{c||}{} & \multicolumn{3}{c|}{ Expansión } & \multicolumn{3}{c||}{ Contracción } \\
\cline { 2 - 7 } & $\begin{array}{c}\text { Retardo } \\
\text { medio } \\
\text { (trimestres) }\end{array}$ & $\begin{array}{c}\text { Duración media } \\
\text { (trimestres) }\end{array}$ & $\begin{array}{c}\text { Amplitud } \\
\text { media } \\
(\%)\end{array}$ & $\begin{array}{c}\text { Retardo } \\
\text { medio } \\
\text { (trimestres) } \\
1\end{array}$ & $\begin{array}{c}\text { Duración } \\
\text { media } \\
\text { (trimestres) }\end{array}$ & $\begin{array}{c}\text { Amplitud } \\
\text { Media } \\
\text { Valor absoluto } \\
(\%)\end{array}$ \\
\hline \hline Trabajadores CS & -6 & 15,50 & 237,60 & $-2,50$ & 10,50 & 193,78 \\
\hline Trabajadores SL & $-4,50$ & 15,50 & 254,47 & $-1,67$ & 8,50 & 270,08 \\
\hline \hline
\end{tabular}

(1) CS, Laborales y ocupados respecto a PIB. + (-) implica retardo (adelanto)

Fuente: BUSY y elaboración propia

Las principales ideas que se desprenden del análisis de la caracterización cíclica realizado, pueden resumirse en los siguientes puntos:

- En general, las fases cíclicas del número de CS tienen mayor duración que las fases del PIB y de las SL, tanto en la expansión como en la contracción.

- En las fases expansivas las SL presentan mayor capacidad para incrementar su número y en las fases contractivas mayor capacidad de supervivencia que las CS.

- La mayor capacidad de generar empleo en las fases expansivas lo presentan las SL, en cambio, en las fases contractivas es el empleo de las CS el que tiene mayor resistencia.

- El empleo de las CS y las SL adelanta sus puntos de giro respecto a los puntos de giro del PIB. Esto es, empiezan a crecer y a decrecer antes que la actividad económica. 
- En general, las fases de las CS evolucionan de forma más desvinculada de la actividad económica española que las fases de las SL. Aspecto que deberá corroborarse con el análisis del grado de sincronización.

\subsection{Sincronización cíclica}

La caracterización de las fases cíclicas descrita afecta sin duda al grado de sincronización con el ciclo económico. A continuación nos ocuparemos de su estudio para ver hasta que punto se produce una relación pro-cíclica o contra-cíclica entre el número de empresas y el número de trabajadores de CS y SL y la actividad económica española.

Para cuantificar el grado de sincronización, el primer índice que se ha calculado es el índice de concordancia (I) que para todo el periodo, alcanza los valores de la tabla 4. Los datos destacan un comportamiento muy ligado al ciclo económico en el número de SL dadas de alta a la Seguridad Social y en su volumen de trabajadores $(0,892$ y 0,757 respectivamente). Estos resultados indicarían que la creación o desaparición de SL sigue el patrón de la actividad económica y acaba trasladándose con menor intensidad a la creación o destrucción de empleo.

Por lo que respecta a los índices entre el PIB y las CS apuntan a un nivel relativamente bajo de sincronización, en esta ocasión más destacable para el número de trabajadores que para el número de empresas $(0,541$ y 0,608 respectivamente). También se constata en la tabla 4 que los índices de simetría entre las CS y las SL son relativamente bajos, tanto en cuanto al número de empresas como a su nivel de empleo (0,581 y 0,649 respectivamente). Se confirma con ello que las CS se mueven al margen del ciclo económico y del propio comportamiento de las SL. Destacar en este sentido que la ocupación de las CS está muy relacionada con la actividad agraria, más del $40 \%$ de la producción final agraria se comercializa a través de estas asociaciones (Gómez, 2004). A su vez, dicha producción está muy condicionada por la aleatoriedad de los factores climatológicos.

Tabla 4: Índice de concordancia

\begin{tabular}{||l|c||}
\hline PIB- número CS & 0,608 \\
\hline PIB- número SL & 0,892 \\
\hline PIB- trabajadores CS & 0,541 \\
\hline PIB- trabajadores SL & 0,757 \\
\hline Número CS-número SL & 0,581 \\
\hline Trabajadores CS-trabajadores SL & 0,649 \\
\hline
\end{tabular}

Fuente: Elaboración propia 
Para alcanzar nuestro segundo objetivo, detectar la existencia de un comportamiento pro/contra-cíclico de las cooperativas y las sociedades laborales, veamos hasta qué punto el grado de simetría/asimetría cíclica es significativo. Para ello estimamos el coeficiente de correlación ( $\rho$ ) usando el método generalizado de momentos. La tabla 5 nos ofrece los valores de la $t$-student y del nivel de significación.

Tabla 5: $t$-Student y significación. Estimación $\rho$

\begin{tabular}{|l|c|c||}
\hline & t-Student & Sign. \\
\hline PIB-Número CS & 0,395664 & 0,6935 \\
\hline PIB-Número SL & 7,371023 & 0,0000 \\
\hline PIB-Trabajadores CS & $-0,239707$ & 0,8112 \\
\hline PIB-Trabajadores SL & 2,532594 & 0,0135 \\
\hline
\end{tabular}

Fuente: Elaboración propia

Los datos de la tabla 5 confirman el carácter pro-cíclico de las SL. La t-student es positiva y significativa en la estimación del coeficiente que relaciona el PIB y las dos variables de las SL. De nuevo se observa como el número de SL presenta una mayor alineación con la actividad económica que el número de trabajadores. Por lo que respecto a las CS, se reafirma su evolución al margen de la actividad económica. Ni el número de CS ni el número de trabajadores alcanzan una estimación significativa de su correlación con el PIB. Es más, el signo de la $t$-student de la estimación entre el PIB y el número de trabajadores es negativo. De lo que se deduce un comportamiento contra-cíclico, aunque no significativo, del número de trabajadores de las cooperativas. Resultado que vendría a avalar un leve efecto refugio del empleo en las cooperativas dentro de los años de estudio.

Para finalizar analizaremos la evolución de la sincronización mediante el índice de asimetría (IA). Los gráficos 1 a 4 muestran el IA dentro del periodo al que se ha ajustado la tendencia lineal estimada. Según el indicador de asimetría, con el paso del tiempo se han agudizado las diferencias entre la actividad económica y las CS (véase la pendiente positiva de los gráficos 1 y 3). En el caso de las SL los gráficos indican un incremento de la simetría entre el PIB y el número de SL (véase pendiente negativa del gráfico 2) y una disminución entre el número de trabajadores de estas empresas y el PIB (véase pendiente positiva gráfico 4). 
Gráfico 1: IA PIB/número CS

Gráfico 2: IA PIB/número SL

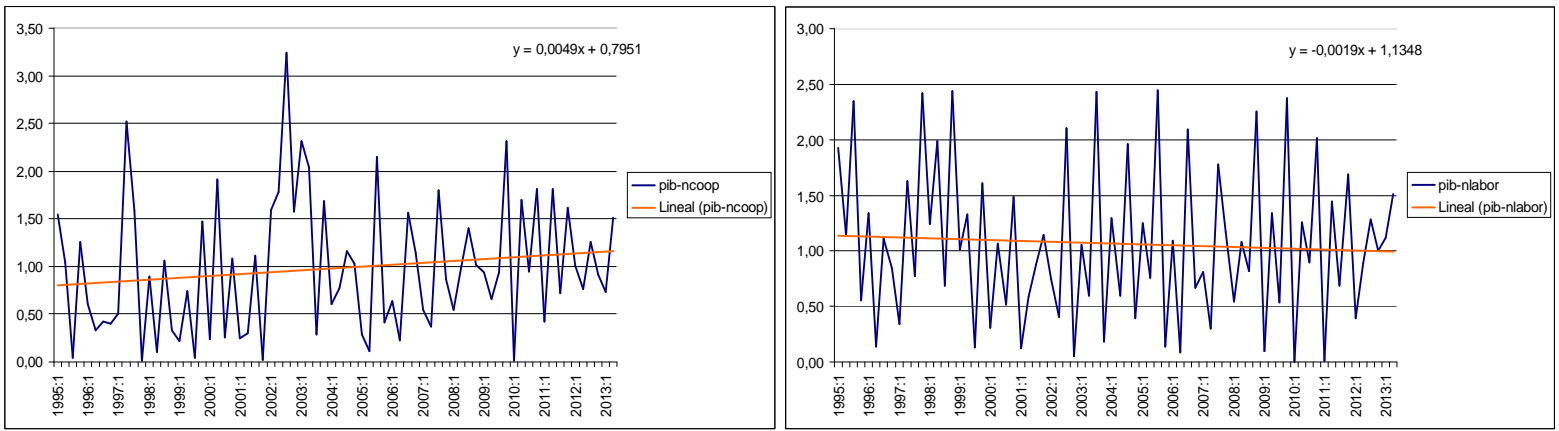

Gráfico 3: IA PIB/Trabajadores CS

Gráfico 4: IA PIB/trabajadores SL
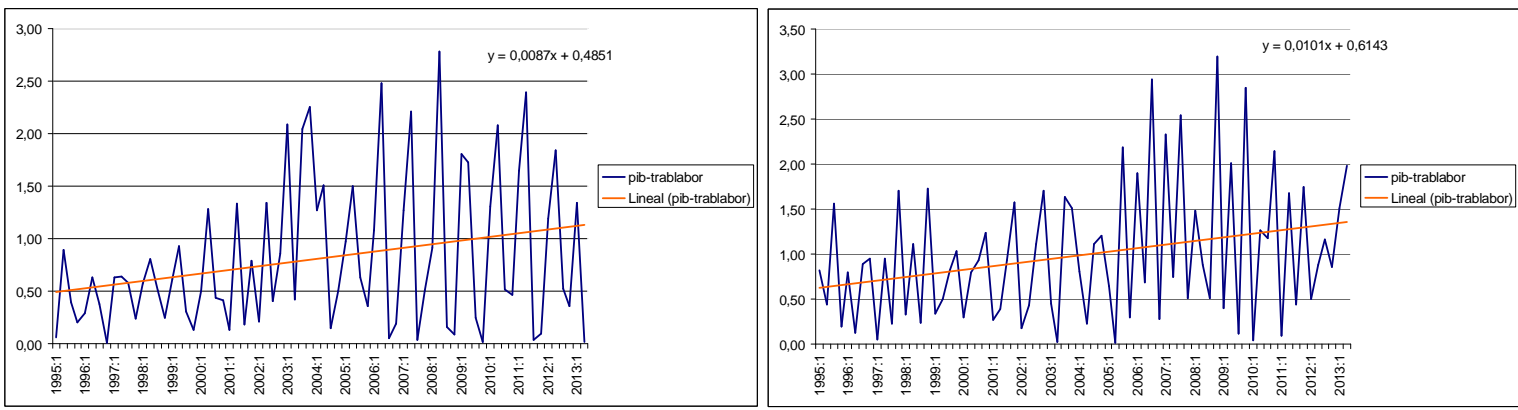

Fuente: Elaboración propia

\subsection{El impacto de la crisis del 2007 en la sincronización cíclica}

El fuerte impacto de la crisis iniciada a mediados del año 2007 nos lleva a preguntar si la tendencia del IA descrita puede estar influenciada por las cifras de los años de crisis. Para responder a esta cuestión, en el anexo recogemos los gráficos del IA dividiendo el periodo en dos subperiodos. Los gráficos de A-1 a A-4 recogen el IA de los trimestres correspondientes a 1995:1-2006:4 y los gráficos A-5 a A-8 los correspondientes al IA de los trimestres correspondientes a 2006:1-2013:2. De su análisis podemos destacar los siguientes aspectos.

Con la crisis se ha agudizado el grado de asimetría entre el ciclo de la actividad económica española y el número de CS (la pendiente del gráfico A-5 es más pronunciada que la del gráfico A-1). Se constata un grado de supervivencia relativamente importante de las CS en el actual escenario de crisis. Asimismo, en los años de crisis ha habido un desacoplamiento cíclico entre el PIB y el número de SL (la pendiente del gráfico A-2 es negativa y las del A-6 es positiva). La crisis ha disminuido el comportamiento pro-cíclico de las SL o dicho de otro modo, en estos años, el grado de resistencia de las SL es relativamente destacable. Se confirmaría la tesis de Grávalos y Pomares (2001a) según la cual en épocas de crisis se 
produce un fenómeno de transformación de empresas tradicionales en empresas de participación con la finalidad de intentar mantener los puestos de trabajo.

Sin embargo, también se observa que la crisis ha incrementado la simetría entre el PIB y el número de trabajadores de ambos tipos de empresas (las pendientes de los gráficos A-3 y A-4 son positivas y las de los A-7 y A-8 son negativas). Así, el empleo de las SL ha incrementado su anclaje al ciclo económico mientras que las CS han reducido su desacoplamiento. La fuerte destrucción de empleo de estos años ha afectado negativamente al comportamiento del empleo en ambos tipos de empresa, a pesar de su remarcable grado de supervivencia. Ni la transformación de empresas tradicionales en empresas de participación, ni la mayor flexibilidad para ajustar el empleo vía horas trabajadas que citan Calderón y Calderón (2012a), han sido suficientes para ejercer un efecto refugio.

Para ver el impacto de la crisis sobre el empleo se ha estimado de nuevo el coeficiente de correlación ( $\rho$ ) para el periodo anterior a la crisis (1995-2006). El resultado es el que recoge el gráfico 5. Aunque sea sólo a nivel informativo, porque la pérdida de datos hace también perder robustez, vemos que sin tener en cuenta la crisis el empleo de las SL sigue siendo pro-cíclico pero no significativamente y el empleo de las CS alcanza un efecto refugio significativo. Lo que de nuevo pone de manifiesto el ya mencionado efecto negativo que ha tenido la crisis sobre el empleo de estas empresas.

Gráfico 5: t-student estimación $\rho$

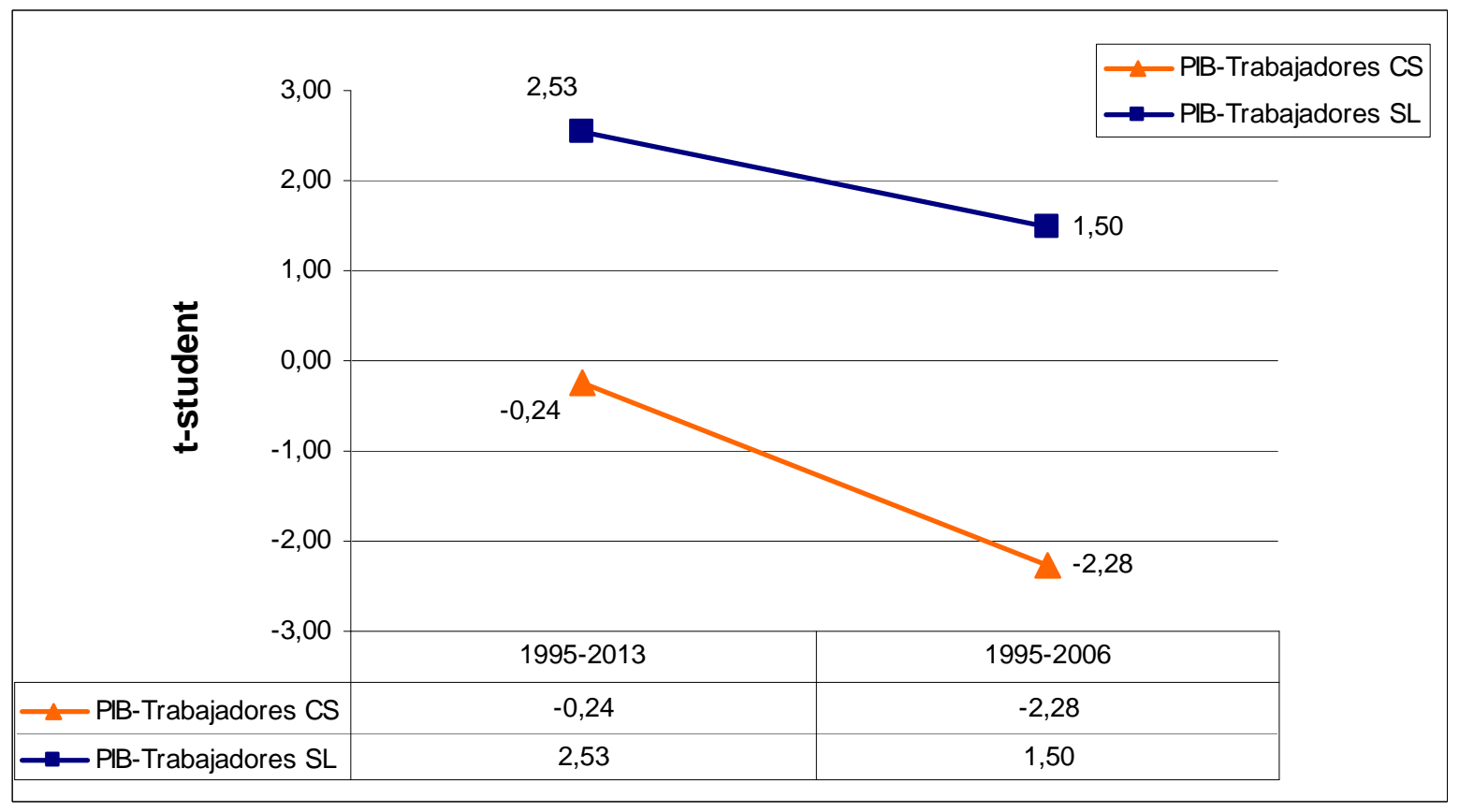

Fuente: Elaboración propia 


\section{CONCLUSIONES}

El trabajo propone caracterizar las fases cíclicas del número de empresas y del empleo de las cooperativas y de las sociedades laborales y contrastar su comportamiento con el del conjunto de la actividad económica española. Asimismo, se ha analizado la incidencia de la crisis iniciada en 2007 en la sincronización de los ciclos. Una de las aportaciones novedosas del estudio es la posibilidad de detectar las diferencias cíclicas entre las dos principales tipologías de empresas de la Economía Social.

En el conjunto del periodo, el comportamiento cíclico de las CS y de las SL es distinto. Por lo que respecta al número de empresas, en las fases expansivas la entrada de SL es relativamente elevada y la pérdida relativamente baja en comparación con las CS. Así, de los resultados se deduce que en las fases expansivas del ciclo, de entre ambas tipologías de empresas se prefiere crear SL. Además para todo el periodo analizado, se ha constatado que resisten mejor los embates de las fases contractivas. Sin embargo, esta capacidad de supervivencia no se traslada al empleo. Si bien en las fases expansivas ganan más empleo que las CS, en las contractivas son las CS las que tienen menos pérdidas de empleo. Las CS tendrían un mayor grado de estabilidad en el empleo que las SL.

Estas diferencias hacen intuir que su relación con el ciclo económico español será divergente. En este sentido, si tenemos en cuenta las dos líneas de trabajo que debaten si las CS muestran un comportamiento contra-cíclico o bien una evolución al margen de las condiciones generales de la economía, los resultados de nuestro trabajo se decantan por la segunda. En efecto, se ha detectado que tanto en las fases del ciclo económico expansivas como en las contractivas, las fases de las CS evolucionan de forma bastante desvinculada de la actividad económica. El número de CS alcanza valores relativamente bajos de sincronización y su coeficiente de correlación con la actividad económica aunque positivo, no es significativo. El empleo de las CS tampoco presenta sincronización con el ciclo económico, aunque el signo negativo del estadístico $t$-student, le acerca a un comportamiento contra-cíclico que sin ser significativo, permite deducir un leve efecto refugio. A conclusiones distintas se llega en el caso de las SL. Estas empresas presentan un comportamiento procíclico significativo, tanto en el caso del número de empresas como en el empleo, aunque mucho más significativo en el número. Por tanto, la creación o desaparición de SL está muy ligado al patrón de la actividad económica y acaba trasladándose, aunque con menor intensidad, a la creación o destrucción de empleo. 
En relación a los efectos de la crisis decir que ha puesto de manifiesto un importante grado de supervivencia de las empresas de la Economía Social. Sin embargo, el empleo se ha visto afectado negativamente. De esta forma se ha constatado que el empleo de las SL está siendo más pro-cíclico y el de las CS ha disminuido su desacoplamiento. Como indican Monzón y Chaves (2012) para la Economía Social en su conjunto, todo parece indicar que las CS y la SL resistieron mejor que el sector privado la primera embestida de la crisis, sin embargo, a partir de 2010 sufrieron pérdidas netas de empleo.

De todas formas el trabajo ha mostrado un comportamiento cíclico de las empresas sociales relativamente más adecuado que el del ciclo económico. En este sentido, en primer lugar, hemos visto que las empresas sociales recuperan la capacidad de generar empleo cuando la actividad económica todavía no ha alcanzado la fase expansiva. En segundo lugar, en las fases contractivas, las SL tienen un elevado grado de resistencia y las CS presentan relativamente menores pérdidas de empleo. Es por ello que nos atrevemos a indicar que parece necesario potenciar este tipo de fórmulas empresariales y de ayudar a los emprendedores sociales a poner en marcha sus proyectos. No cabe duda que es una de las vías a tener en cuenta para salir de las crisis.

\section{BIBLIOGRAFÍA}

BEN-NER, A. (1988) The life -cycle of worker-owned firms in market economies. A theorical analysis Journal of Economic Behavior and Organization, 10 (3), p. 287-313.

BRY, G. y BOSCHAN, Ch. (1971) Cyclical analysis of time series: selected procedures and computer programmes. New York, National bureau of Economic Research.

BURNS, A. F., y W. C. MITCHELL (1946) Measuring Business Cycles. NBER, Studies in Business Cycle, Columbia University Press, New York.

CALDERÓN, B. y CALDERÓN, M.J. (2012a) La calidad del empleo de las entidades de la economía social en el período de crisis. Ekonomiaz, 79, p. 30-57.

CALDERÓN, B. y CALDERÓN, M.J. (2012b) Cómo afrontar la crisis las cooperativas en España: comparativa de trayectorias laborales a partir de la Muestra Continua de Vidas Laborales. CIRIEC-España, Revista de Economía Pública, Social y Cooperativa, 76, p. 526.

CECOOP-CICOPA Europe (The European Confederation of Cooperatives and worker-owned enterprises active in industries and services) (2013) Business Transfers to Employees under the Form of a Cooperative in Europe. Opportunities and Challenges. 
CLEMENTE, J.; DÍAZ, M. y MARCUELLO, C. (2008) Estudio sobre las cooperativas y sociedades laborales en España: creación de empleo y contribución al desarrollo económico, Proyecto de Investigación, memoria Final, Dirección General de la Economía Social, del trabajo autónomo y del Fondo social Europeo, Ministerio de Trabajo e Inmigración.

CLEMENTE, J.; DÍAZ, M. y MARCUELlO, C. (2009) Sociedades cooperativas y sociedades laborales en España: estudio de su contribución a la creación de empleo y al crecimiento económico. REVESCO. Revista de Estudios Cooperativos, 98, p. 35-69.

DÍAZ, M. y MARCUELLO, C. (2010) Impacto económico de las cooperativas. La generación de empleo en las sociedades cooperativas y su relación con el PIB. CIRIECEspaña Revista de Economía Pública, social y Cooperativa, 67, p. 23-44.

DEMOUSTIER, D. (2000) Análisis del empleo. Capítulo II, en Chaves, R. Economía Social y empleo en la Unión Europea, CIRIEC-España, p. 48-82.

FIORENTINI, G. y PLANAS, CH. (2003) User manual BUSY-Program. EC Fifth Framework Program, Joint Research Centre of the European Commission, Ispra.

GADEA, M.D.; GÓMEZ-LOSCOS, A. y MONTAÑ́ES, A. (2012) Cycles incide cycles: Spanish regional aggregation. Series, 3 (4), p. 423-456.

GÓMEZ, J.D. (2004) La reforma de la PAC y la importancia de las cooperativas agrarias en la vertebración socioeconómica y territorial del medio rural. ERÍA, 63, p. 73-90.

GRÁVALOS, M.A. y POMARES, I. (2001a) Cooperativas, desempleo y efecto refugio. REVESCO. Revista de Estudios Cooperativos, 74, p. 69-84.

GRÁVALOS, M.A. y POMARES, I. (2001b) La adaptación de las sociedades laborales a la evolución del ciclo económico. Un estudio empírico par alas diferentes comunidades autónomas. Revista de Economía Pública, Social y Cooperativa, CIRIEC-España, 38, p. $33-55$.

HAMILTON, J. (1989) A new approach to the economic analysis of nonstationary time series and the business cycle. Econometrica 57 (2), p. 357-384.

HARDING, D. y PAGAN, A. (2002) Dissecting the Cycle: a Methodological Investigation. Journal of Monetary Economics, 49, p. 365-381.

HARDING, D. y PAGAN, A. (2006) Synchronization of cycles. Journal of Econometrics,132 (1): p. 59-79.

HASSLER, J, (2003) Är Sverige I takt med Europa? en Ulf Jakobsson (ed.), Därför euron-tio ekonomer om den gemensamma valutan, Ekerlids Förlag, Stockholm. 
LARSSON, A.; GACO, N. y SIKSTRÖM, H. (2009) Aggregate and regional business cycle synchronization in the Nordic countries. Swedish Institute for European Policy Studies Sieps), Report $n^{\circ} 7$.

LEJARRIAGA, G.; BEL, P. y MARTÍN, S. (2013) El emprendimiento colectivo como salida laboral de los jóvenes: análisis del caso de las empresas de trabajo asociado. REVESCO. Revista de Estudios Cooperativos, 112, Extraordinario en Homenaje al Profesor Alfonso Carlos MORALES, p. 36-65.

LEVIN, H. (1984) Employment and productivity of producer cooperatives. EN: Ed. R. Jackall y H. Levin, Worker cooperatives in America Berkeley: University of California P, p. 88106.

MELIÁN, A. y CAMPOS, V. (2010) Emprendedurismo y Economía Social como mecanismo de inserción sociolaboral en tiempos de crisis. REVESCO. Revista de Estudios Cooperativos, 100, p. 43-67.

MONZÓN, J.L. (1991) Análisis del empleo en la Economía Social. Revista de Economía y Sociología del Trabajo, 12, p.17-24.

MONZÓN, J.L. (2012) Las cooperativas ante la globalización: magnitudes, actividades y tendencias. Ekonomiaz, 79, p. 13-30.

MONZÓN, J.L. y CHAVES, R. (2012) La Economía Social en la Unión Europea. Informe elaborado para el Comité Económico y social europeo por el CIRIEC.

NEWEY, W.K. y WEST, K.D. (1987): A simple, positive semi-definite, heteroskedasticity and autocorrelation consistent covariance matrix, Econometrica, 55 (3), p.703-708.

PENCAVEL, J.; PISTAFERRI, L. y SCHIVARDI, F. (2006): Wages, Employment, and Capital in Capitalist and Worker-Owned Firms. Industrial and Labor Relations Review, 60 (1), p. 23-44.

PÉROTIN, V. (2006) Entry, exit, and the business cycle: Are cooperatives different? Journal of Comparative Economics, 34 (2), p. 295-316.

ROMÁN, C. (2010) Las cooperativas españolas y los ciclos económicos: una primera aproximación, 1942-2002. Documento de Trabajo 103 de la Asociación española de historia Económica (AEHE),

RUSSELL, R. y HANNEMAN, R. (1992) Cooperatives and the business cycle: The Israeli case. Journal of Comparative Economics, 16, p. 701-715.

STABER, U. (1993) Worker cooperatives and the business cycle: are cooperatives the answer to unemployment? The American journal of economics and sociology, 52 (2), p. 129-143. 
TARAZONA, P. y ALBORS, J. (2005) La economía social y el desarrollo local. Revista Nacional del CIDEC, $\mathrm{n}^{\circ} 45$, p. 70-75.

\section{ANEXO}

\section{6:1-2006:4}

Gráfico A-1: IA PIB/Número CS

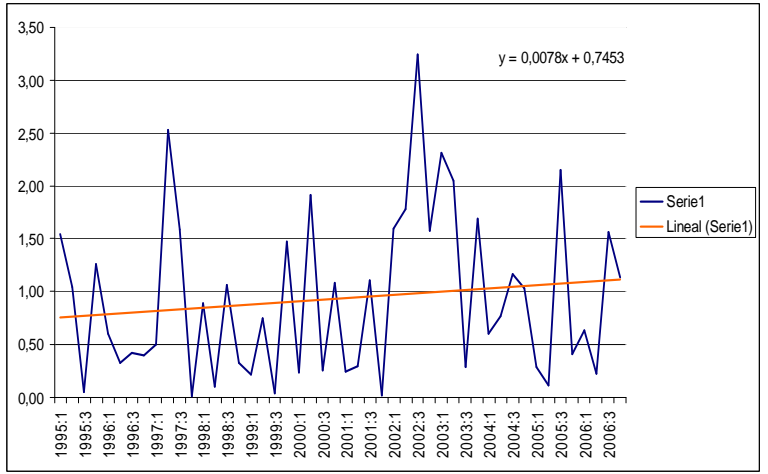

Gráfico A-2: IA PIB/Número SL

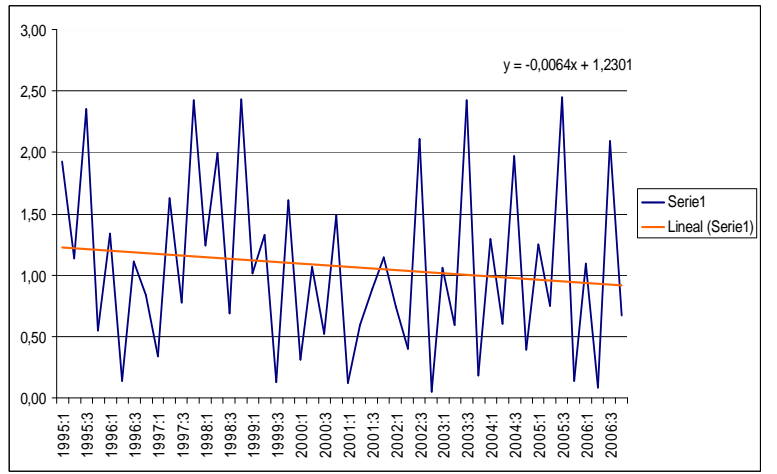

Gráfico A-3: IA PIB/Trabajadores CS Gráfico A-4: IA PIB/Trabajadores SL
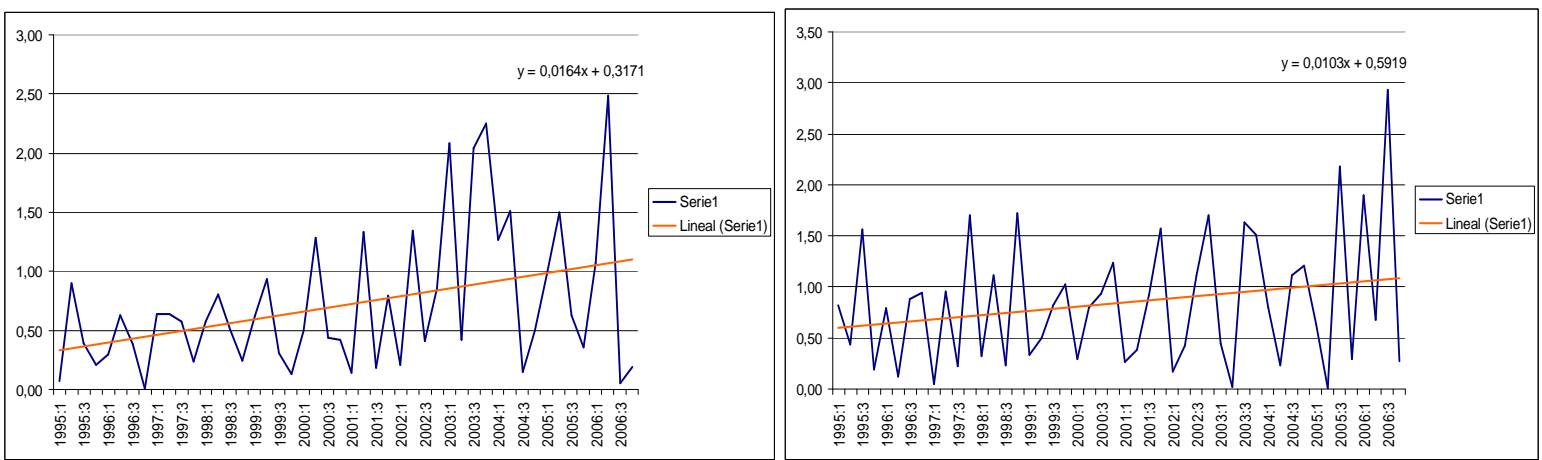

2006:1-2013:2

Gráfico A-5: IA PIB/Número CS

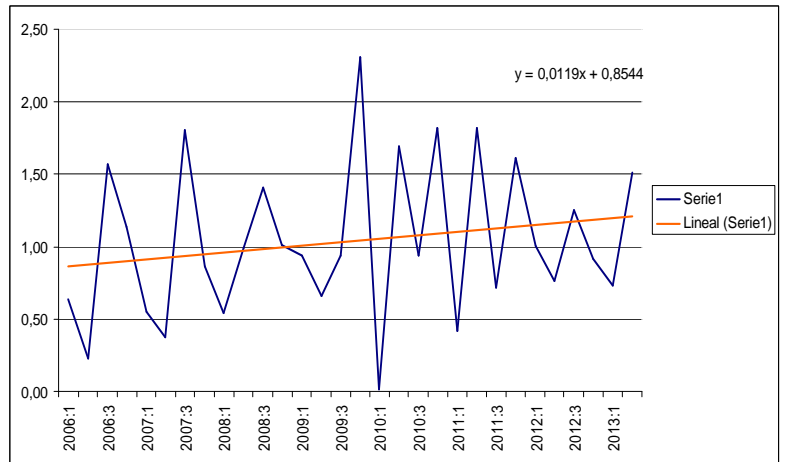

Gráfico A-6: IA PIB/Número SL

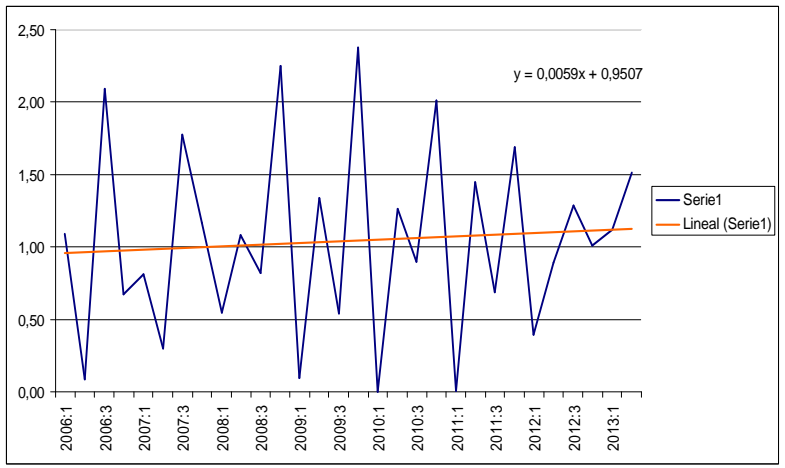


Gráfico A-7: IA PIB/Trabajadores CS Gráfico A-8: IA PIB/Trabajadores SL

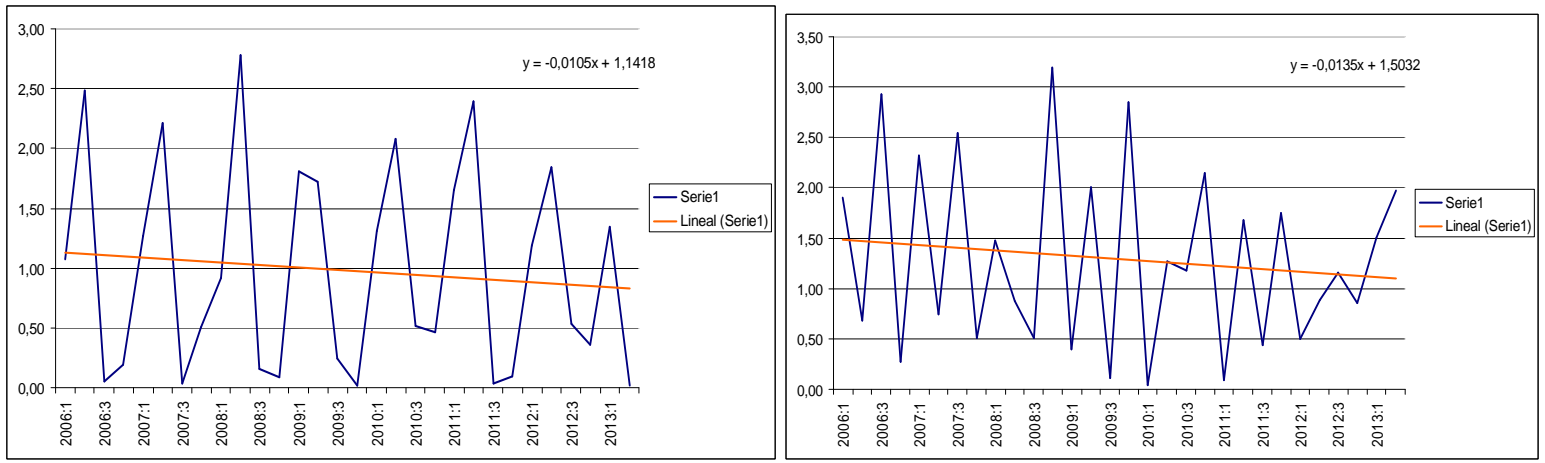

Fuente: Elaboración propia 